\title{
Parents Concern Regarding Dental Treatment for Children During COVID-19 - A Survey
}

\section{Meenapriya M늘 Deepa Gurunathan², Deepika Rajendran ${ }^{3}$}

\section{ABSTRACT}

Background: COVID-19 [Coronavirus] was declared as a pandemic disease by the World Health Organization in the year 2020. Almost all the dental care facilities in the affected countries were closed and provided only minimal dental services. Several guidelines were proposed by the government for providing essential and non-essential dental services. Several parents were not accessible to the dentists and faced difficulties in providing treatment for their children. The dental emergencies among the child include trauma, severe dental caries, pulpitis, and swellings. Treatment should be provided regarding these dental emergencies.

Aim: The aim of this study is to assess the difficulties faced by the parent for dental treatment in their child during COVID-19.

Materials and Methods: This study was conducted through an online questionnaire during pandemic disease COVID-19. The survey was circulated among the parents, whose child required dental treatment and this study assessed the difficulties faced by parents regarding dental treatment for their children. The data were recorded and tabulated.

Results: The mean age of the parents who took part in this study was $38.85 .50 .59 \%$ were females and $49.41 \%$ were males. The mean age of the child whose parents were involved in this study was $10.52 .91 .95 \%$ of the patients were aware of the COVID-19 condition. $27.59 \%$ of the parents encountered dental problems in their children. $35.9 \%$ of the parents consulted the dentists regarding the dental problem encountered in their children. Various treatment options preferred by the parents were going to the dentists [50.91\%], online consultations [27.27\%], and Home remedy [21.82\%]. 59.46\% of the children were relieved from their pain after the treatment. $43.24 \%$ of the parents found that online consultations were effective and satisfying. $58.62 \%$ of the parents have the fear of transmission during the dental visits for their child. Reasons for not accessible to the dentists among parents were Transportation problems [72\%], Dentists were not available [24\%], other reasons [4\%]. 58.62\% of the parents were aware of the essential and non-essential dental treatment.

Conclusion: The difficulties faced by the parents in the treatment of their child during COVID-19 were most commonly associated with the transportation problem. Most of the parents preferred home remedies due to the fear of the pandemic situation. The prevalence of dental emergencies among children is comparatively lesser. Awareness about the essential and non-essential dental treatment prevails.

Key Words: COVID-19, Parents, Dental treatment, Child, Emergencies, Dentists

\section{INTRODUCTION}

COVID-19 is an infectious pandemic disease caused by the Coronavirus. This was declared as a pandemic disease by the World Health Organization in 2020. The virus that causes COVID-19 transmits through the droplets of the infected person through cough, sneeze, or exhales. These droplets take the medium of air and fall on the floors or surfaces. Coronavirus disease 2019 became a major public health concern affecting the world. This concern started in China and spread to other parts of the world. ${ }^{1}$ The novel Coronavirus belongs to a family of single-stranded RNA viruses, which are known as Coronaviridae. ${ }^{2}$ These families of viruses are zoonotic and transmitted from animals to humans. They include severe acute respiratory syndrome coronavirus. These viruses were found abundantly in the nasopharyngeal and salivary secretions of the affected persons. Dentists, who encounter infections of the oral cavity, had higher chances of COVID-19 transmission. Appropriate health management oral disease prevention was carried out by the government to prevent the rapid spread of the virus.

\section{Corresponding Author:}

Deepa Gurunathan, Professor, Department of Pedodontics, Saveetha Dental College, Saveetha Institute of Medical and Technical Sciences, Saveetha University, Chennai, 162, Poonamallee High Road, Chennai 600077, Tamil Nadu, India; Telephone: +91 99946 19386; Email: deepag@saveetha.com

ISSN: 2231-2196 (Print)

Received: 27.07 .2020
ISSN: $0975-5241$ (Online)

Revised: 28.08 .2020
Accepted: 24.09 .2020
Published: 20.10 .2020 
The most common dental concerns encountered in a child are trauma, dental caries associated with pain, dental abscess, early childhood caries, canker sores, malalignment, and sensitive teeth. Children and the adolescents frequently come to the dental clinic in the emergency department for the treatment of their child's dental emergencies. ${ }^{3}$ Many of these visits are related to the traumatic injuries. The knowledge of the general dentists about these traumatic injuries prevails, but there was a lack of consistency. ${ }^{4}$ However, previous literature studies in other parts of the world reveal, unrelated to trauma, the other emergency conditions to treat will be the dental caries. Most of dental emergencies are related to dental caries. ${ }^{5}$ Significant dental caries may slow down the child's growth and that after receiving the dental treatment. The salivary MDA levels are found to be higher in patients with early childhood caries. This can be suppressed by the use of antioxidants. ${ }^{6}$ The fluoride level of water is an important tool in preventing caries. ${ }^{7}$ The use of appropriate fluoridated toothpaste also plays a role in preventing dental caries. ${ }^{8}$ Access to preventive and emergency dental care has become an area of increasing concern. ${ }^{9}$

Pediatric patients, who required the dental treatment, were not able to access the dentists due to various reasons during the COVID-19 pandemic condition. Due to the lockdown of the entire country, parents found difficulty in accessing the dentists due to a lack of public transport facilities. Several guidelines were proposed by the government for the public due to COVID-19 and restricted the public from coming out of their houses. The complications associated with untreated dental concerns in children would be severe pain, tooth abscess, and acute dental infections. The frenal attachment and the morphology of it vary among populations and with age. ${ }^{10}$ These can affect the aesthetics of the child and can be a concern of the parent. This would be the non-essential dental treatment of children during COVID-19. The implications of dental care provided during this COVID-19 were provided. 11

Previous research conducted on the parent's concern regarding dental treatment during pandemic conditions revealed that the outbreak of COVID-19 has a great impact on the parents where if there is need for a dental emergency, parents prefer to stay home and use home remedies. ${ }^{12}$ Few dental clinics provide emergency dental care during COVID-19. ${ }^{13}$ The reasons for not visiting the dentists and the awareness of the parents about the essential and non-essential dental treatment were given by previous literature. The use of dental services among the parents for their child is most common only during cases of emergencies. ${ }^{14,15}$

This study brings about the importance of emergency dental visits during pandemic COVID-19. Emergency dental care providers were accessible, and they were advised to follow the guidelines by IDA. ${ }^{16}$ The various essential and non-es- sential dental treatments have been enlisted and awareness was provided to the public. ${ }^{17}$ The various reasons for the parents not able to access the dentists, include curfew and transportation during COVID-19. This study fulfills the need for dental visits during pandemic COVID-19.

Therefore, this study aims at the difficulties faced by the parents to access the dentists during COVID-19.

\section{MATERIALS AND METHODS}

This study is a questionnaire study conducted among the parents during pandemic COVID-19 caused by noble coronavirus 2019.

\section{ONLINE QUESTIONNAIRE}

PARENTS CONCERN REGARDING DENTAL TREATMENT FOR CHILDREN DURING COVID 19

1) Your age (age of the parent)

2) Gender

3) Age of your child

4) Gender of your child

5) Are you aware of the recent COVID 19 condition? Yes No

6) Are you aware that COVID 19 is pandemic? Yes No

7) Did you encounter any dental problem in your child [pain, swelling, etc.,] during this lockdown period? Yes No

8) If yes, did you consult with the dentists for the treatment of the same?

Yes No

9) If no, reason for not consulting the dentists for the treatment of your child?

Pain was tolerable

Any other reasons - specify

10) Among these, which one did you prefer for dental treatment of your child?

- Going to the dentists

- Staying at home, using home remedy

- Online consultations

11) Did your child relieve from the dental problem after you followed any of the above options?

Yes No

12) Age of your child who needed dental treatment?

13) If you had visited the dentist regarding the dental treatment for your child, were you denied treatment? Yes No

14) If you had preferred for online consultations, were you able to follow up with the dentists and satisfied with the treatment for the problem?

Yes No

15) Reasons for not visiting the dentists during this period? 
- Fear of transmission

- Any others - specify

16) Were you able to access the dentists in case of dental emergencies [ severe pain, pus, swelling, etc.,] Yes No

17) If yes, did you have the fear of current COVID 19 transmission while visiting the dentists?

Yes No

18) If you were accessible to the dentists, specify the following

Government hospitals

Private Institutions

Private hospitals

19) If no, specify the reason, why were you not able to access dentists in case of dental emergencies of your child?

- Transportation problem

- Dentists weren't available

- Any other reasons - specify

20) Are you aware of the essential and non-essential dental treatment?

Yes No

21) In future, if you encounter a dental emergency situation in your child during this period, which one do you prefer for the treatment of the same?

Home remedy

Visiting the dentist

Online consultation

Any others - specify

22) Did you come across any child among your relatives or any other person you know, who were not able to access to the dentists?

Yes

No

23) If yes, specify the age.

This study was conducted in 2020. The study had the advantages of easier data collection due to the online questionnaire circulated. The survey was conducted only among the specific population and various reasons for the parents not able to access the dentists were established. Ethical Approval to conduct this study was obtained from the Institutional Scientific Review Board, Saveetha Dental College and Hospitals.

The survey was conducted among the parents whose children required dental treatment during COVID-19. An online questionnaire was prepared and circulated. The link for the questionnaire - [https://forms.gle/q5wnw8u1dQhTRgzx8 ]. Measures taken to minimize the sampling bias were done. This study was correlated with other parts of the population and the study population was clearly identified. The questionnaire was framed in a simple manner and easily understandable by everyone. Hence, accurate results were provided. The validity of this questionnaire was provided by approval from the authors. Since a randomized sampling was done among the general public, the study results can be generalized to the South Indian population. The pandemic COVID-19 is applicable to all parts of the population.

For the data collection, this questionnaire was circulated through google forms. The data were collected and distribution was done according to various responses by the parents. The validity and reliability of this study were assessed. The incomplete data which included the inappropriate responses were managed by the exclusion of the same.

This online questionnaire study was analyzed. The independent variables include the availability of dentists and Transportation. The major reasons for not accessing dentists were noted. The dependent variables include the reasons for visiting the dentists.

\section{Statistical analysis}

These responses were analyzed and data was collected. The data was tabulated in SPSS version 20. The descriptive analysis of this survey was done along with the causal analysis and the graphs were prepared based upon the responses.

\section{RESULTS AND DISCUSSION}

The mean age of the parents who took part in this study is 38.85. $50.59 \%$ of the parents who took part in this study were females and $49.41 \%$ were males. The age of the parents who took part in this study was tabulated in Graph 1. The gender distribution of the parents who were involved in this study was depicted in Graph 2. The mean age of the child, whose parents were involved in this study were 10.52. Most of the age groups involved were 12 years. This is depicted in Graph 3. The parents who were aware of the COVID-19 condition were $91.95 \% .3 .45 \%$ of the parents were not aware of the COVID-19 condition. This graph is depicted in Graph 4. Awareness of the parents that COVID-19 is pandemic revealed that $88.5 \%$ of the parents were aware and $5.75 \%$ of the parents were not aware. [Graph 5]

$27.59 \%$ of the parents encountered dental problems in their children during the COVID-19 lockdown. [Graph 6] Consultation of the parents with the dentists was done by $35.9 \%$ of the parents. $64.1 \%$ of the parents did not consult the dentists for the treatment of the same. [Graph 7] The various reasons for not visiting the dentists regarding the dental concern of their child included that the pain was tolerable [19.77\%], afraid of COVID-19 [1.2\%]. [Graph 8]

The various treatment options preferred by the parents regarding dental concern for their child were going to the dentists [50.91\%], home remedies [21.82\%], online consultations with the dentists [27.27\%]. [Graph 9] 59.46\% of the children found relief of pain after the treatment. $16.2 \%$ of the child experienced pain relief after dental treatment. [Graph $10] 11.49 \%$ of the parents reveal that they were denied treat- 
ment during COVID-19 by the dentists. [Graph 11] 43.24\% of the parents find that the online consultations were satisfying and effective in the treatment of dental concern in their child. [Graph 12]

$58.62 \%$ of the parents have the fear of transmission while visiting the dentists. [Graph 13] The treatments preferred by parents during COVID-19 were in private hospitals [53.19\%], government hospitals [25.53\%], and private institutions [21.26\%]. [Graph 16] The reasons for not being able to access the dentists during lockdown included transportation problems [72\%], dentists were not available [24\%], and other reasons were 4\%. [Graph 17] Awareness of the parents about the essential and non-essential dental treatment includes that $58.62 \%$ of the parents were aware and $16.09 \%$ of the parents were not aware. [Graph 18] The gender of the parent and their awareness of COVID-19 is depicted in graph 19.

The dental treatment options preferred by the parents and their effectiveness in treating the symptoms were depicted in Graph 20. The treatment options preferred by the parents and its outcome are depicted in Graph 21. The parents who did not consult the dentists regarding the dental concern of their child and its correlation with the fear of transmission are illustrated in Graph 22. The dental problems encountered during the lockdown and the treatment options preferred by the parents were depicted in Graph 23.

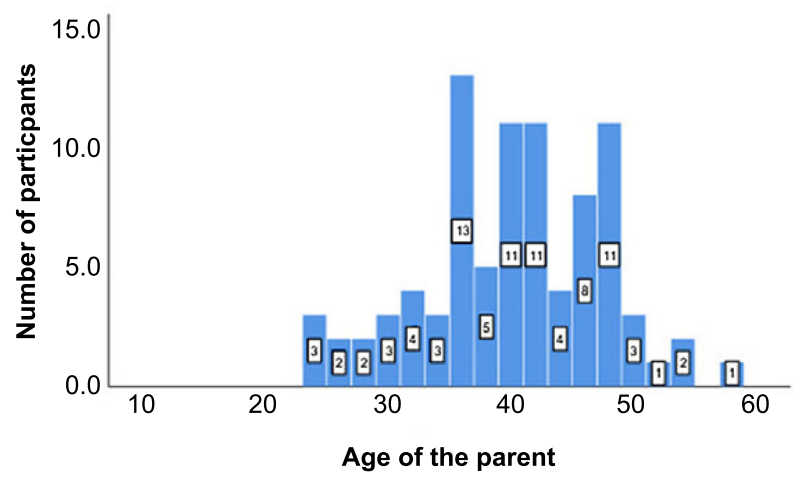

Graph 1: Bar graph showing the age of the parent and the number of participants who participated in this study. [Blue graph shows the different age groups] $\mathrm{X}$-axis shows the age of the parent and $Y$-axis shows the number of participants involved in this study. The highest number of participants was from the age of 34 .

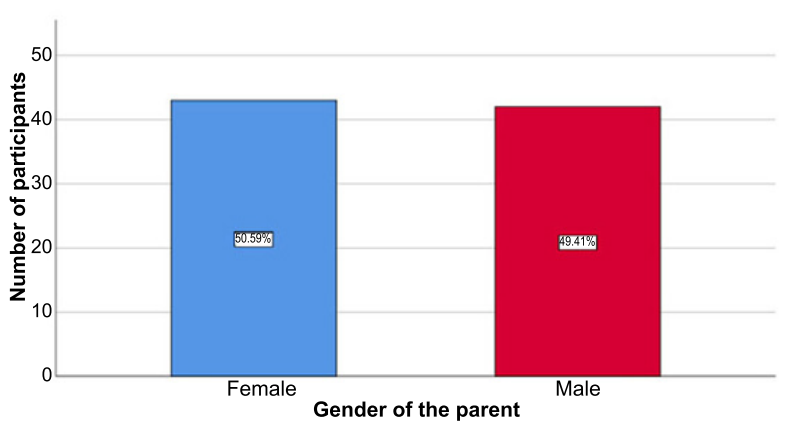

Graph 2: Bar graph showing the gender of the parent and the number of participants who took part in this study. [Blue graph depicts females, red- males] $X$-axis shows the gender of the parent and $\mathrm{Y}$-axis shows the number of participants involved in this study. Female participants were higher [50.59\%] than the males $[49.41 \%]$.

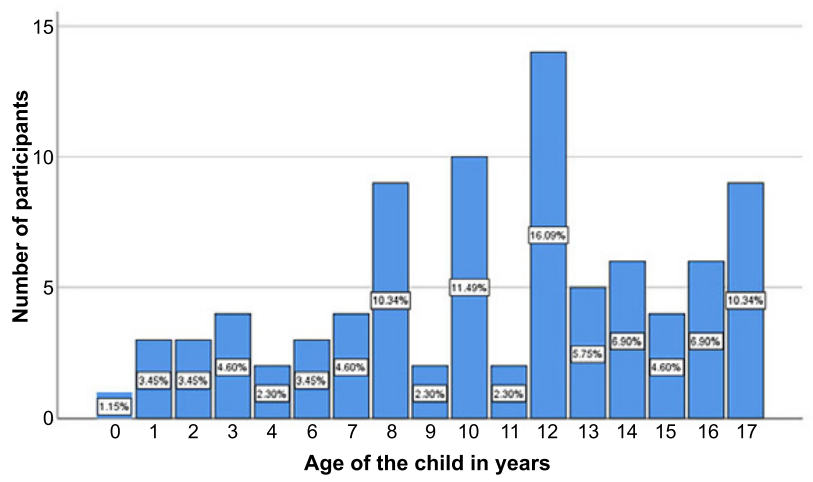

Graph 3: Bar graph showing the age of the child whose parents were involved in this study. [depicted as a blue color for different age] $\mathrm{X}$-axis shows the age of the child and $\mathrm{Y}$-axis shows the number of participants involved in this study. The highest number of participants was from age of 12 years [16.09\%].

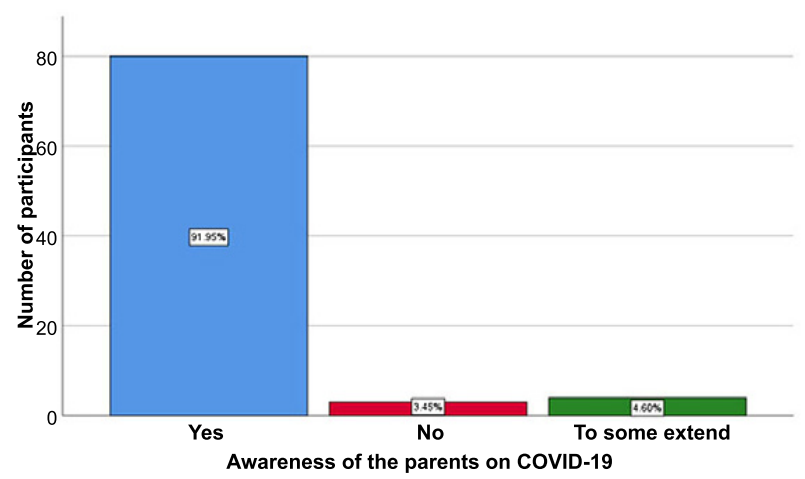

Graph 4: Bar graph showing awareness of the parents on the recent COVID-19 condition. [Blue graph denotes yes, red- no and green -to some extent] $X$-axis shows the awareness of parents on COVID-19 and Y-axis shows the number of participants involved. Most number of participants was aware of COVID-19 [91.95\%]. 


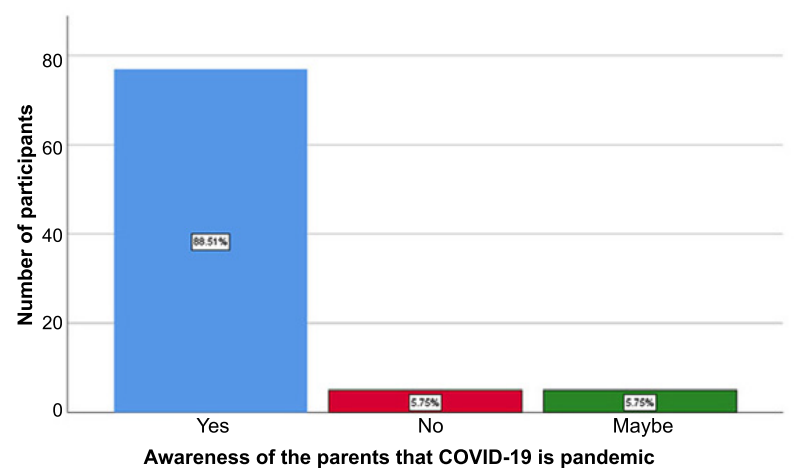

Graph 5: Bar graph showing awareness of the parents that COVID-19 is pandemic. [Blue graph denotes yes, red-no, green-maybe] $\mathrm{X}$-axis shows the awareness of the parents that COVID-19 is pandemic and Y-axis shows the number of participants involved. Most parents were aware of the pandemic condition. [98.51\%].

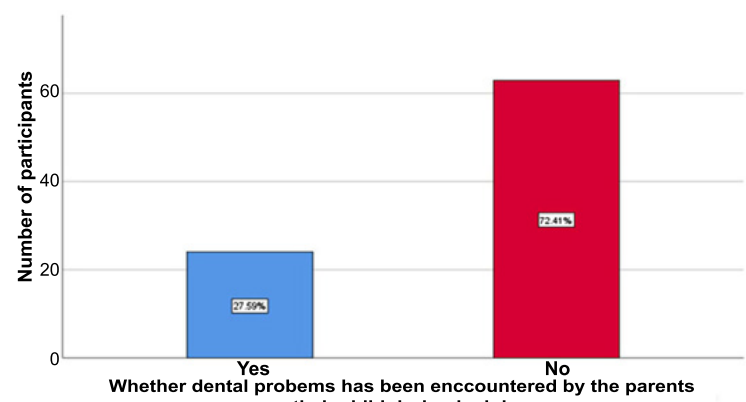

Graph 6: Bar graph showing whether dental problems have been encountered by the parents on their child during the lockdown. [Blue graph denotes yes, red-no] X-axis shows whether a child had dental problems during the lockdown and Y-axis shows the number of participants. $72.41 \%$ of the parents did not encounter dental problems in their child and $27.59 \%$ of the parents encountered dental problems.

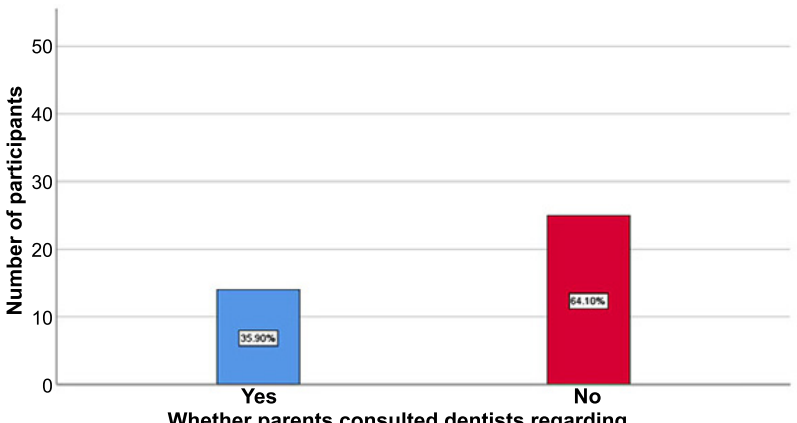

Graph 7: Bar graph showing whether parents consulted dentists regarding dental concerns in their child. [Blue graph denotes yes, red-no] X-axis shows whether parents visited dentists and Y-axis shows the number of participants who visited and did not visit. The graph reveals that $64.1 \%$ of the parents did not visit the dentist and $35.90 \%$ of the parents visited the dentists for the dental problem of their child.

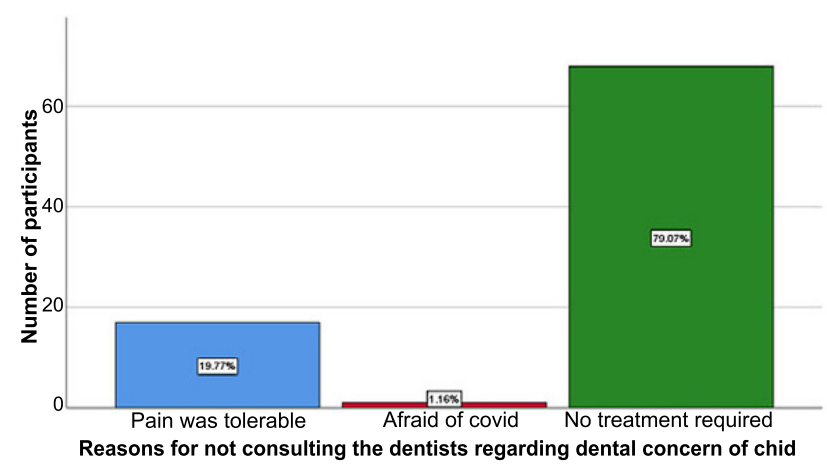

Graph 8: Bar graph showing reasons for not consulting the dentists regarding the dental concern of their child. [Blue graph depicts that the pain was tolerable, red- afraid of COVID, green- no treatment required] X-axis shows the reasons for not consulting the dentists regarding the dental concern of their child and Y-axis shows the number of participants involved. $19.77 \%$ of the parents reveal that the pain was tolerable and $1.2 \%$ of the parents were afraid of COVID-19.

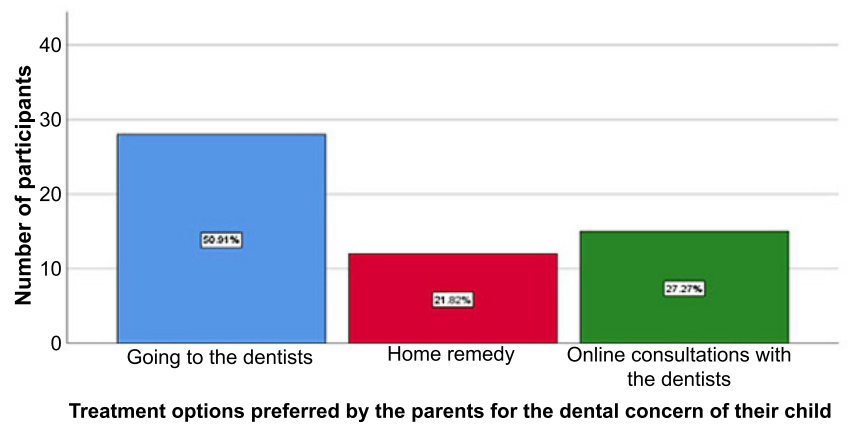

Graph 9: Bar graph showing treatment options preferred by the parents for the dental concern of their child. [Blue graph depicts going to the dentists, red-home remedy, green-online consultations with the dentists] $50.91 \%$ of the parents preferred going to the dentists and $21.82 \%$ of the parents prefer home remedy. $27.27 \%$ of parents prefer online consultations.

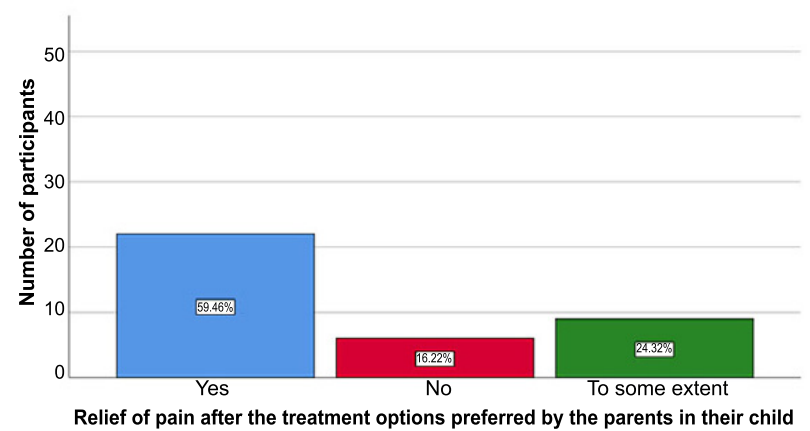

Graph 10: Bar graph showing the effectiveness of the treatment option preferred by the parent. [Blue graph denotes yes, red- no, green- to some extent] $X$-axis shows whether a child 
has been relieved from pain after treatment and $\mathrm{Y}$-axis shows the number of participants involved. $59.46 \%$ were relieved of pain and $16.22 \%$ of the children were not relieved of pain following the treatment.

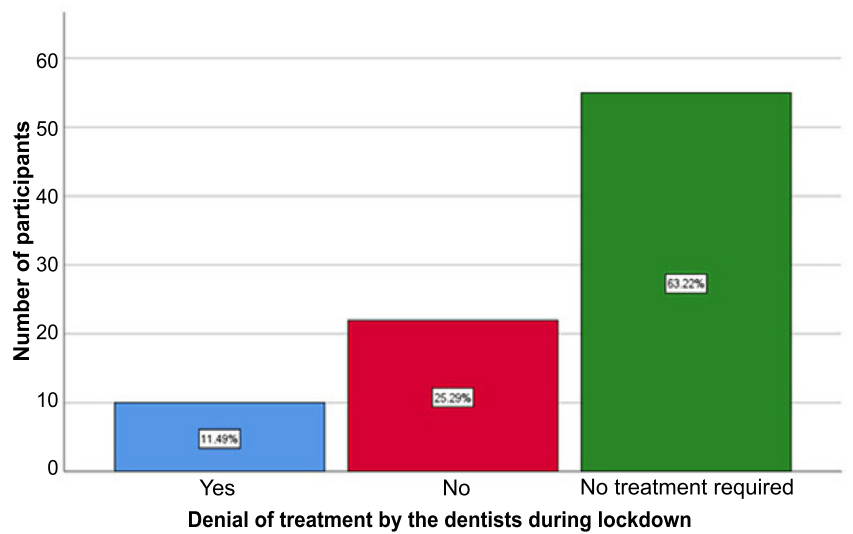

Graph 11: Bar graph showing a denial of treatment by the dentists for their patients with dental problems. [Blue graph depicts yes, red- no, green- no treatment required] $X$-axis shows whether dentists denied treatment for patients and $Y$ axis shows the number of participants involved. $11.49 \%$ of the dentists denied treatment during the lockdown and $25.29 \%$ of the dentists did not deny treatment.

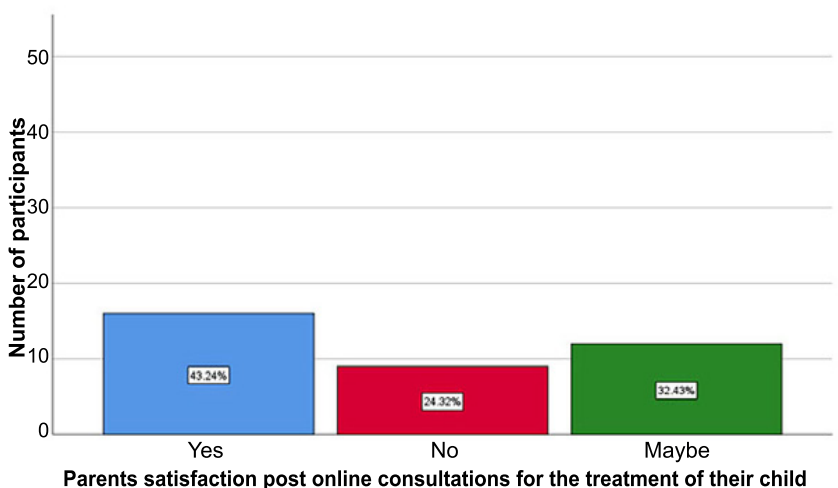

Graph 12: Bar graph showing the effectiveness of online consultations for the dental concern of the child. [Blue graph denotes yes, red- no, green - maybe] $43.24 \%$ of the parents found online consultations as effective for the dental concern of child, and $24.32 \%$ found online consultations as ineffective.

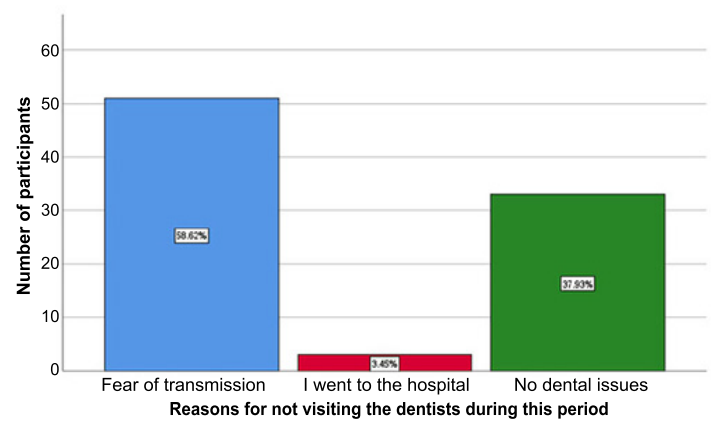

Graph 13: Bar graph showing reasons for parents not consulting with the dentists during the lockdown. [Blue graph denotes fear of transmission, red- people who visited dentist, green-no dental issues] $X$-axis shows the reasons for not visiting the dentists and $\mathrm{Y}$-axis shows the number of participants involved in this study. $58.62 \%$ of the parents had fear of transmission whereas $3.45 \%$ of the parents visited the dentists.

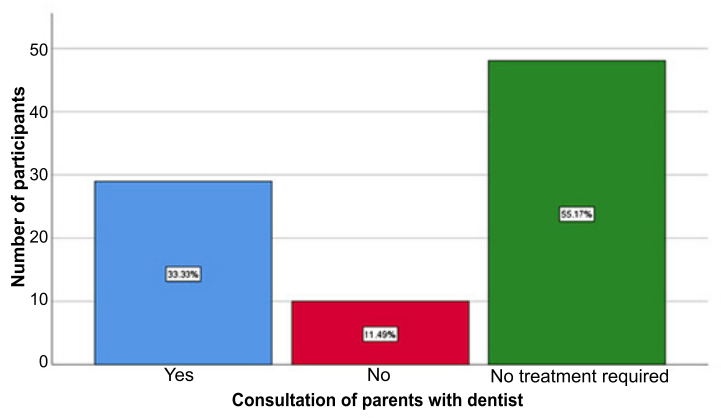

Graph 14: Bar graph showing whether parents were able to access dentists during COVID-19 lockdown. [Blue graph denotes yes, red-no, green-no treatment required] $X$-axis shows whether parents were able to access the dentists and Y-axis shows the number of participants involved. $33.33 \%$ were accessible to dentists and $11.49 \%$ of the parents were not accessible.

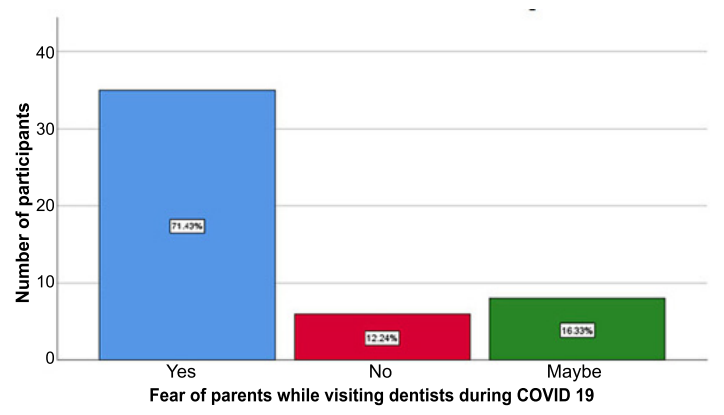

Graph 15: Bar graph showing whether parents had fear of COVID-19 transmission while visiting dentists regarding dental treatment for their child. [blue graph denotes yes, red-no, green-maybe] $\mathrm{X}$-axis shows the fear of parents while visiting dentists during COVID-19 and Y-axis shows the number of participants involved in this study. $71.43 \%$ of the parents had the fear of transmission than $12.24 \%$ of the parents who did not have fear. 


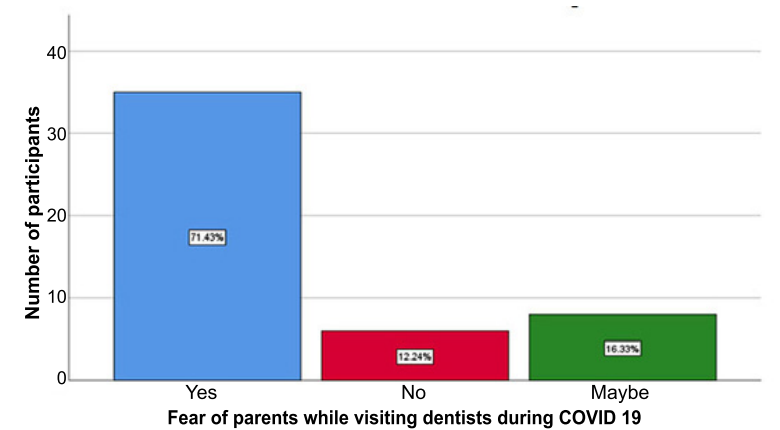

Graph 16: Bar graph showing hospitals or institutions preferred by parents for the treatment of their child. [Blue graph depicts government hospital, red-private hospital, green-private institutions] Most of the parents preferred private hospitals for the treatment of their child [53.19\%] than the government hospital [25.53\%] and private institutions [21.26\%].

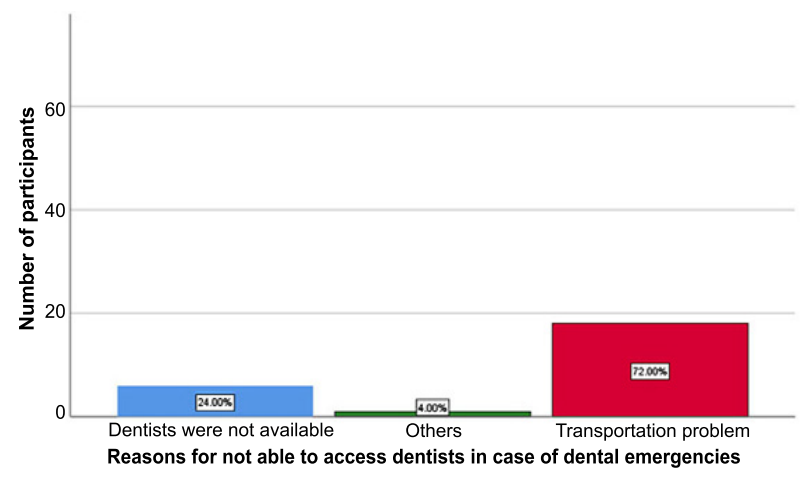

Graph 17: Bar graph showing reasons why the parents were not able to access dentists during COVID-19 lockdown. [Blue graph depicts that the dentists were not available, red- transportation problem, green-others] Most of the parents found transportation problems as a reason for not able to access dentists [72\%] and $24 \%$ reported that dentists were not available during the lockdown.

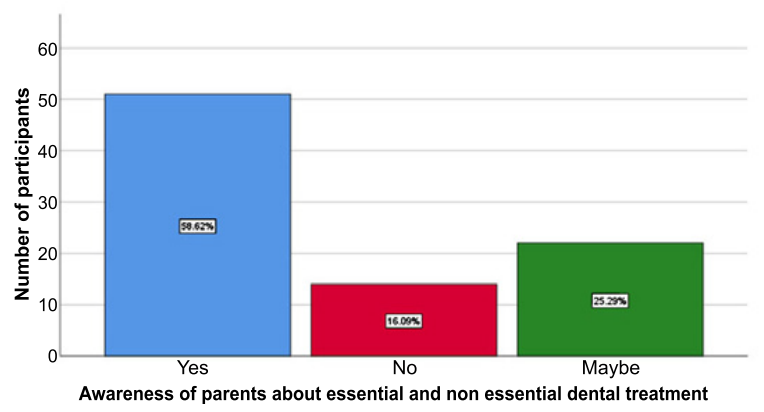

Graph 18: Bar graph showing awareness of parents about essential and non-essential dental treatment. [Blue graph denotes yes, red- no, green- maybe] $X$-axis shows the awareness of parents about essential and non-essential treatment and Y-axis shows the number of participants involved in this study. $58.62 \%$ of the parents were aware and $16.09 \%$ of the parents were not aware of essential and non-essential treatments.

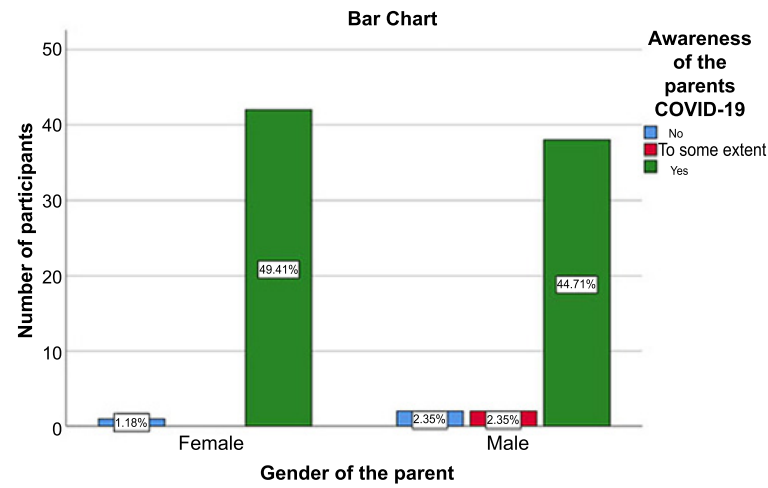

Graph 19: Bar graph showing the gender of the parent and their awareness on COVID-19. [Blue color depicts no, greenyes, red-to some extent] $X$-axis shows the gender of the parent and Y-axis shows their awareness about COVID-19. Both females and females there was no difference in awareness of parents of COVID-19 between males and females. However, this is not statistically significant (Pearson's Chi-square test; $\mathrm{p}$-value $=0.183-$ not significant)

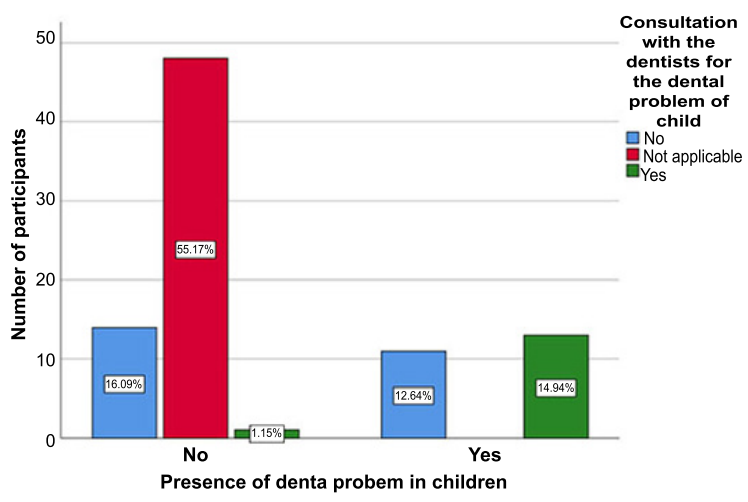

Graph 20: Bar graph showing dental problems encountered by the parents during COVID-19 lockdown and whether they consulted with dentists regarding the treatment of the same. [Blue graph depicts no, red- not applicable, green- yes] Xaxis shows the presence of dental problems of the child and Y-axis shows whether they consulted the dentists regarding the treatment of the same. Most of the parents consulted dentists [14.94\%] when there was a presence of dental problems, compared to $12.64 \%$ of parents who did not visit the dentists even though they had dental problems. However, this was statistically significant (Pearson's Chi-square test; $p$-value $=0.00$ - significant). 


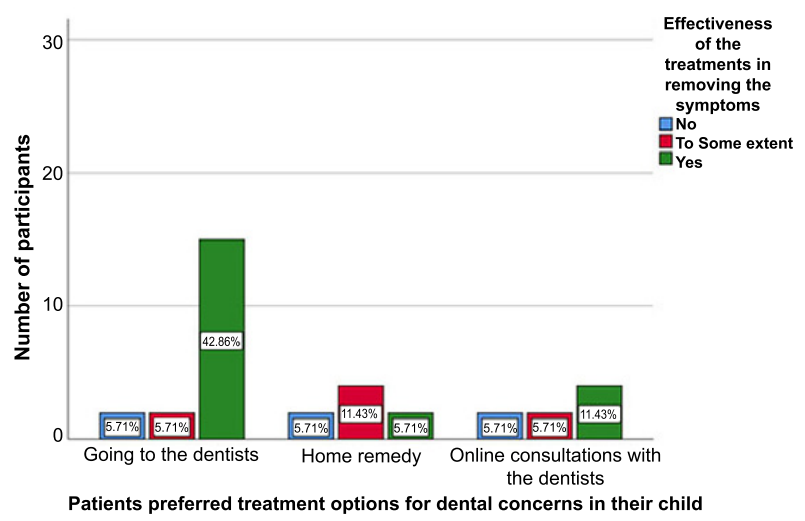

Graph 21: Bar graph showing preferred treatment options by the parents for their child and its effectiveness in treating the symptoms. [Blue graph denotes no, green- to some extent, orange-yes] $\mathrm{X}$-axis shows the preferred treatments by the parents for the dental problem in their child and Y-axis shows the effectiveness following the treatment preferred. The effectiveness of the treatment in removing the symptoms was higher when patients preferred to visit the dentist [42\%] while it was to some extent when they tried home remedies [11.43\%]. However, this was statistically significant (Pearson's Chi-square test; p-value $=0.00$ - significant) .

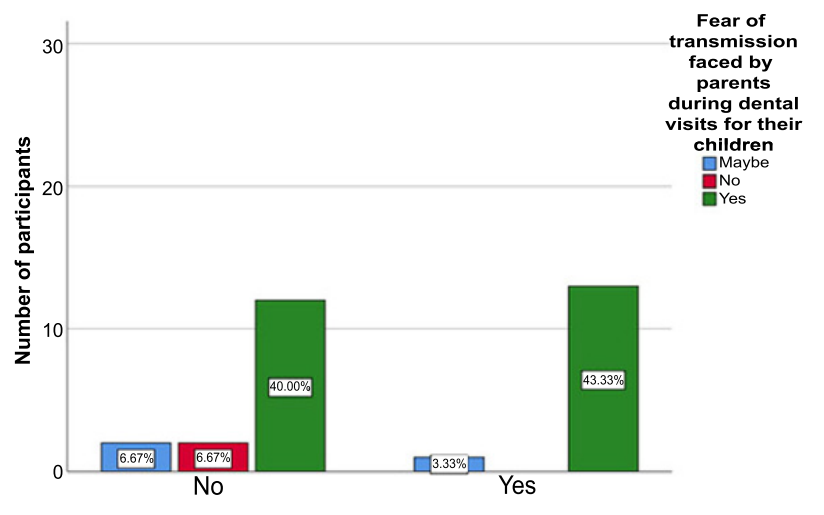

Consultation with the dentists during dental problem of child

Graph 22: Bar graph showing parents who were able to access dentists during COVID-19 and their fear about its transmission. [Blue graph denotes maybe, red-no, orange-yes] $X$-axis shows the parents who consulted the dentists for the dental concern of their child. Y-axis shows the fear of transmission during their visit. There was no difference in the fear of transmission of COVID-19 in patients who visited dentists [43.3\%] and patients who didn't visit dentists for the dental concern of their child [40\%]. However, this was statistically significant (Pearson's Chi-square test; $p$-value $=0.00$ - significant).

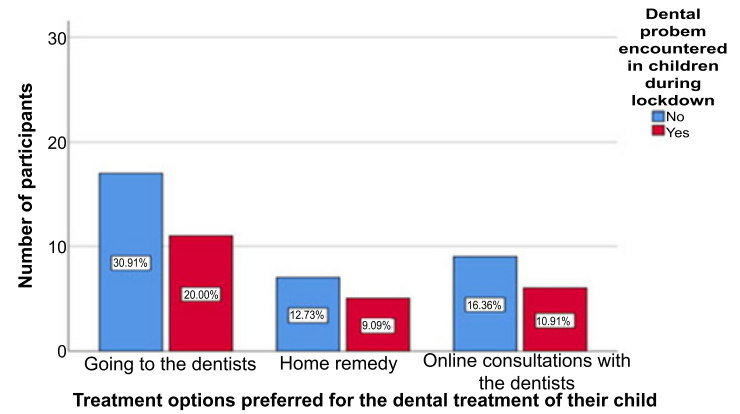

Graph 23: Bar graph showing dental problems encountered by the child and the various treatment options preferred by parents during the lockdown. [Blue graph denotes- no, redyes] $X$-axis shows the children who had dental problems and Y-axis shows the various treatment options preferred by the parents. A higher number of parents preferred going to the dentists [20\%] even when they didn't have dental concerns than the parents who had the dental concern in their child [30\%]. However, this was statistically significant (Pearson's Chi-square test; $p$-value $=0.004$-significant).

The highest age group of the parents who took part in this study was 30 to 40 years. The reason for this could be that the age of the child whose parents took part in this study was 0 to 17 years. The gender distribution of the parents who took part in this study was equal. The inclusion criteria for this study are parents and there is an equal gender prevalence of males and females. The most common age group of the child whose parents were involved in this study was 12 . This is because the age groups included in this study were from 0 to 17 years. All the participants were aged from 0 to 17 years. Most of the people were aware of the COVID-19 condition and its pandemic situation. The reasons for this increased awareness are due to mass communication. Governments and the media are spreading increased awareness among the public. $^{18,19}$

The dental problems encountered by the parents in their child were lesser. The reason for this could be that the prevalence of dental emergencies being lesser among children. Not all the child undergoes dental emergencies. ${ }^{20}$ The parents mostly did not consult the dentists regarding dental treatment for their child. This is due to the fear of transmission due to pandemic COVID-19. ${ }^{21}$ Difficulties in accessing the dentists were experienced.

Most participants revealed that the pain was tolerable and that was the reason for not consulting dentists during the lockdown. Most of the parents prefer the home remedies for the dental treatment of their child due to the fear of transmission. ${ }^{22}$ Some of the parents preferred going to the dentists comparatively. The reasons could be due to its effectiveness. Most of the patients were relieved from the pain after treatment options preferred by the parents in their child. Analgesics and antibiotics play a role in dental emergencies. The 
denial of treatment by the dentists was comparatively lesser. The dentists have higher rates of transmission of COVID-19 during dental treatments. This could be the possible reason for the denial of treatment. ${ }^{23}$ Few clinics are open for dental emergencies.

A higher number of participants were satisfied with the online consultations. Since it is easy to access and the treatment options are preferred by the dentists based on photographs. Lesser chances of transmission are prevailing through online consultations. ${ }^{24}$ Fear of transmission of the parents during dental visits is higher. Previous literature provides the fear of transmission as the reason for the parents not visiting the dentists. ${ }^{25}$ Due to the recent COVID-19 evolution, no supporting studies were available sufficiently.

Most participants were able to access dentists in case of emergencies. However, the transportation problem prevails to a greater extent. This is due to the restrictions, public transportations, and lockdown situation. The awareness about the essential and non-essential dental treatment prevails to a greater extent among the parents. The possible reason is due to mass communication, media in spreading awareness among the public.

Since this study was conducted in 2020, the pandemic situation was new and no sufficient supporting and contradictory studies are available.

The previous studies were conducted on the treatment of severe dental caries such as pulpitis through pulpectomy with the use of kedo files. The study reported increased quality of obturation with lesser duration. ${ }^{26}$ Since, the treatment takes only less time, these can be used during these pandemic situations for faster procedures. Previous study reports the less duration time of kedo files than the conventional files. ${ }^{27,28}$ Kedo files also serve better than the rotary files and the reciprocating files. ${ }^{29}$ Similarly, the use of rotary files can reduce the treatment duration with increased quality of obturation. ${ }^{30}$ The knowledge about the use of these rotary files such as the protaper gold, etc. was assessed. The study reported that more awareness should be conducted to emphasize the increased advantages of the rotary files. ${ }^{31}$ The rotary files when compared with the manual instrumentation had similar quality of obturation but better in less duration of the rotary files. ${ }^{32}$

Ranula is a lesion that occurs on the floor of the mouth. It can cause difficulty in breathing when it extends into the pharyngeal space. This ranula can turn out to be a dental emergency when it becomes severe. ${ }^{33}$ These types of emergencies can also occur in the pandemic conditions and facilities should be provided. Dental neglect is another condition where the child is unaware, shows neglect towards the dental treatment, and their oral hygiene considerations are poor. ${ }^{34}$ In these patients' chewable toothbrush will serve as the best alternative to the manual toothbrushes. ${ }^{35}$

\section{Limitations}

The limitations of this study include convenience sampling of patients. Limited and specific population was covered. The management aspect was not conducted.

\section{Future Studies}

This study should be conducted with large sample size. A large area of the population should be covered. The management strategies taken by the parent should be considered. The dentists should be given awareness about the prevalence of dental emergencies.

\section{CONCLUSION}

Within the limits of this study, the prevalence of dental emergencies among children is less. Parents who met with dental problems in their child found that consultation with the dentists was useful in treating the symptoms. When asked for the preference for dental treatment during the lockdown, the parents found online consultations were effective and safe. The majority of parents showed fear towards COVID-19 and also, the same as the reason for not visiting dentists. Transportation problem plays a significant role in accessing dentists. Awareness about the COVID-19 condition and the essential and non-essential dental treatment prevails among the parents. The dentists should be therefore be given awareness about the percentage of prevalence of dental emergencies.

\section{ACKNOWLEDGEMENT}

This study was supported by Saveetha Dental College and Hospitals, Saveetha Institute of Medical and Technical Sciences, Saveetha University, Chennai.

\section{Conflict of Interest}

There were no conflicts of interest as declared by the authors.

\section{Financial Support}

Self-funding

\section{REFERENCES}

1. Wang Y, Zhou C-C, Shu R, Zou J. [Oral Health Management of Children during the Epidemic Period of Coronavirus Disease 2019]. Sichuan Da Xue Xue Bao Yi Xue Ban. 2020 Mar;51(2):151-4.

2. Ather A, Patel B, Ruparel NB, Diogenes A, Hargreaves KM. Coronavirus Disease 19 (COVID-19): Implications for Clinical Dental Care. J Endod. 2020 May;46(5):584-95.

3. Dorfman DH, Kastner B, Vinci RJ. Dental concerns unrelated to trauma in the pediatric emergency department: barriers to care. Arch Pediatr Adolesc Med. 2001 Jun;155(6):699-703.

4. Ravikumar D, Jeevanandan G, Subramanian EMG. Evaluation of knowledge among general dentists in treatment of traumatic 
injuries in primary teeth: A cross-sectional questionnaire study. Eur J Dent. 2017 Apr;11(2):232-7.

5. Wilson S, Smith GA, Preisch J, Casamassimo PS. Nontraumatic dental emergencies in a pediatric emergency department. Clin Pediatr. 1997 Jun;36(6):333-7.

6. Subramanyam D, Gurunathan D, Gaayathri R, Vishnu Priya V. Comparative evaluation of salivary malondialdehyde levels as a marker of lipid peroxidation in early childhood caries. Eur J Dent. 2018 Jan;12(1):67-70.

7. Somasundaram S, Ravi K, Rajapandian K, Gurunathan D. Fluoride Content of Bottled Drinking Water in Chennai, Tamilnadu. J Clin Diagn Res. 2015 Oct;9(10):ZC32-4.

8. Mahesh R MM. Fluoride, Fluoridated Toothpaste efficacy and its safety in children- a review. International Journal of Pharmaceutical Research. 2018;10(4):109-14.

9. Acs G, Shulman R, Ng MW, Chussid S. The effect of dental rehabilitation on the body weight of children with early childhood caries. Pediatr Dent. 1999 Mar;21(2):109-13.

10. Linda Christabel S, Gurunathan D. Prevalence of Type of Frenal Attachment and Morphology of Frenum in Children, Chennai, Tamil Nadu. World Journal of Dentistry. 2015 Oct 1;6(4):203-7.

11. Zhou P, Yang X-L, Wang X-G, Hu B, Zhang L, Zhang W, et al. A pneumonia outbreak associated with a new coronavirus of probable bat origin. Nature. 2020 Mar;579(7798):270-3.

12. Karimi M. Coronavirus (COVID-19) and Fear of Pediatric Dental Treatment. EC Paediatrics. 2020 May;9(5).

13. Rambabu T, Koneru S. Reasons for use and nonuse of dental services among people visiting a dental hospital in urban India: A descriptive study. J Educ Health Promot. 2018 Aug 2; 7:99.

14. Heaton LJ, Smith TA, Raybould TP. Factors influencing use of dental services in rural and urban communities: considerations for practitioners in underserved areas. J Dent Educ. 2004 Oct;68(10):1081-9.

15. Luzzi V, Ierardo G, Bossù M, Polimeni A. COVID-19: Pediatric Oral Health During and After the Pandemics. MEDICINE \& PHARMACOLOGY. 2020. Available from: https://www. preprints.org/manuscript/202004.0002/v1. Accessed on June 11 2020.

16. Zeng L, Su T, Huang L. Strategic plan for management in oral and maxillofacial surgery during COVID-19 epidemic. Oral Oncol. 2020 Jun; 105:104715.

17. Hammel JM, Fischel J. Dental Emergencies. Emerg Med Clin North Am. 2019 Feb;37(1):81-93.

18. Mahase E. China coronavirus: what do we know so far? BMJ. 2020 Jan 24;368:m308. Available from: http://dx.doi. org/10.1136/bmj.m308. Accessed on June 112020.

19. Li RWK, Leung KWC, Sun FCS, Samaranayake LP. Severe Acute Respiratory Syndrome (SARS) and the GDP. Part I: Epidemiology, virology, pathology and general health issues. $\mathrm{Br}$ Dent J. 2004 Jul 24;197(2):77-80.

20. Alharbi A, Alharbi S, Alqaidi S. Guidelines for dental care provision during the COVID-19 pandemic. Saudi Dent J. 2020 Apr 7;

21. Bai Y, Yao L, Wei T, Tian F, Jin D-Y, Chen L, et al. Presumed Asymptomatic Carrier Transmission of COVID-19. JAMA. 2020 Feb 21;

22. Chen H, Guo J, Wang C, Luo F, Yu X, Zhang W, et al. Clinical characteristics and intrauterine vertical transmission potential of
COVID-19 infection in nine pregnant women: A retrospective review of medical records. Lancet. 2020 Mar 7;395(10226):80915.

23. Day M. Covid-19: ibuprofen should not be used for managing symptoms, say doctors and scientists. BMJ. 2020 Mar 17;368:m1086.

24. Volpato LER, Palti DG, Lima JE de O, Machado MA de AM, Aranha AMF, Bandeca MC, et al. When and Why Parents Seek Dental Care for Children under 36 Months. J Int Oral Health. 2013 Aug;5(4):21-5.

25. Rowley ST, Sheller B, Williams BJ, Mancl L. Utilization of a hospital for treatment of pediatric dental emergencies. Pediatr Dent. 2006 Jan;28(1):10-7.

26. Jeevanandan G. Kedo-S Paediatric Rotary Files for Root Canal Preparation in Primary Teeth - Case Report. J Clin Diagn Res. 2017 Mar;11(3): ZR03-5.

27. Jeevanandan G, Govindaraju L. Clinical comparison of Kedo-S paediatric rotary files vs manual instrumentation for root canal preparation in primary molars: a double blinded randomised clinical trial. Eur Arch Paediatr Dent. 2018 Aug;19(4):273-8.

28. Panchal V, Jeevanandan G, Subramanian E. Comparison of instrumentation time and obturation quality between hand K-file, $\mathrm{H}$-files, and rotary Kedo-S in root canal treatment of primary teeth: A randomized controlled trial. J Indian Soc Pedod Prev Dent. 2019 Jan;37(1):75-9.

29. Nair M, Jeevanandan G, Vignesh R, Subramanian EMG. Comparative evaluation of post-operative pain after pulpectomy with $\mathrm{k}$-files, kedo-s files and mtwo files in deciduous molars -a randomized clinical trial. Brazilian Dental Science. 2018; 21:411.

30. Govindaraju L, Jeevanandan G, Subramanian EMG. Comparison of quality of obturation and instrumentation time using hand files and two rotary file systems in primary molars: A single-blinded randomized controlled trial. Eur J Dent. 2017 Jul;11(3):376-9.

31. Govindaraju L, Jeevanandan G, Subramanian EMG. Knowledge and practice of rotary instrumentation in primary teeth among indian dentists: A questionnaire survey. Journal of International Oral Health. 2017 Mar 1;9(2):45.

32. Govindaraju L, Jeevanandan G, Subramanian E. Clinical Evaluation of Quality of Obturation and Instrumentation Time using Two Modified Rotary File Systems with Manual Instrumentation in Primary Teeth. J Clin Diagn Res. 2017 Sep;11(9): ZC55-8.

33. Packiri S, Gurunathan D, Selvarasu K. Management of Paediatric Oral Ranula: A Systematic Review. J Clin Diagn Res. 2017 Sep;11(9): ZE06-9.

34. Gurunathan D, Shanmugaavel AK. Dental neglect among children in Chennai. J Indian Soc Pedod Prev Dent. 2016 Oct;34(4):364-9.

35. Govindaraju L, Gurunathan D. Effectiveness of Chewable Tooth Brush in Children-A Prospective Clinical Study. J Clin Diagn Res. 2017 Mar;11(3): ZC31-4.

36. Manhas S, MDS, Dentistry PAP, Varanasi, Pradesh U. Obturating Materials in Pediatric Dentistry : Literature Review. Vol. 11, International Journal of Contemporary Research and Review. 2020. Available from: http://dx.doi.org/10.15520/ijcrr. v11i08.835. Accessed on 29 August 2020. 